\title{
Temperature and Pressure transducer based on FBG for large diameter water pipes
}

\author{
Antonio Quintela*a ${ }^{*}$ Maria J. Pallol ${ }^{\mathrm{b}}$, Hany S. Roufael ${ }^{\mathrm{a}}$, Oscar Martínez ${ }^{\mathrm{b}}$, José D. San Emeterio ${ }^{\mathrm{b}}$, \\ José M. López-Higuera ${ }^{a}$ \\ ${ }^{a}$ Grupo de Ingeniería Fotónica, Universidad de Cantabria, Ed. I+D+i Telecomunicación, Avda. Los Castros, S/N, 39005, Santander \\ Spain, \\ ${ }^{\mathrm{b}}$ COPSESA S.A. C/ Alcalde Domingo Gómez Maza, S/N, 39800, Ramales de la Victoria, Spain.
}

\begin{abstract}
A specific temperature and pressure optical fiber transducer is presented in this paper. By using a customized fiber reinforced plastic membrane with embedded Fiber Bragg Gratings, the fluid pressure and temperatures changes are converted in optical wavelength displacements. The membrane and the transducer custom design allows a suitable measurand discrimination. The transducer is implemented, characterized and calibrated. Its feasibility to be used on large diameter water pipes has been successfully validated by means of field trials. Many of these transducers will be optically multiplexed to monitoring these infrastructures.
\end{abstract}

Keywords: Transducer, pressure, temperature, fiber Bragg grating, optical fiber.

\section{INTRODUCTION}

The measurement of both pressure and temperature of fluids or gasses in a given technological process is an essential aspect in many industrial applications; being more important when they are working under severe environmental conditions. The measurement of fluid pressure can serve purposes such as strengthen the security of the process, ensuring optimum operating conditions and even monitor in real time any process. This is the case of the transportation of very high fluxes of water using large diameter pipes.

Optical fiber sensors based on Fiber Bragg Gratings (FBG) exhibit advantages such as: chemical and electromagnetic passivity (EMI), biocompatibility, high sensibilities and precisions and what is key advantage for this application: they can be optically multiplexed enabling the construction of sensors networks with a high number of sensing elements[1,2].

Pressure sensors using fiber Bragg gratings (FBG) have been previously reported. However, they are not offering the required technical characteristics to work properly in the specific above mentioned applications. In some cases because their sensitivities are low [3] , or even due to their complex design for manufacturing [3,4], among other reasons.

The conception, design, implementation and characterization of a pressure and temperature transducer using two FBGs properly embedded into a fiber reinforced plastic (FRP) membrane and, also, the field trial results are presented in this paper.

\section{TRANSDUCER}

To reach the final transducer architecture several configurations based on the integration of two FBGs and a membrane have been conceived. All proposals were analyzed taking into account the required technical behaviors including their suitability to be easily fabricated at low cost. Membranes with FBGs placed symmetrically at both sides [6] in such a way that one is elongated (placed on the convex side) and the other is compressed (placed on the concave side) with pressure were successfully checked to obtain pressure sensors insensitive to temperature. However, as both temperature and strain (pressure) were required after several proposals, the final configuration selected is the illustrated in Figure 1. One of the integrated FBG's in the membrane responds with high sensitivity to pressure (the one placed in the middle), being the other practically insensitive to pressure ( placed at the border side). It is worth noting that both are sensitive to temperature.

*quintela@unican.es; phone +34942 206736; fax +34942200877

23rd International Conference on Optical Fibre Sensors, edited by José Miguel López-Higuera,

Julian Jones, Manuel López-Amo, José Luis Santos, Proc. of SPIE Vol. 9157, 91570R

(C) 2014 SPIE · CCC code: $0277-786 X / 14 / \$ 18 \cdot$ doi: $10.1117 / 12.2058765$ 


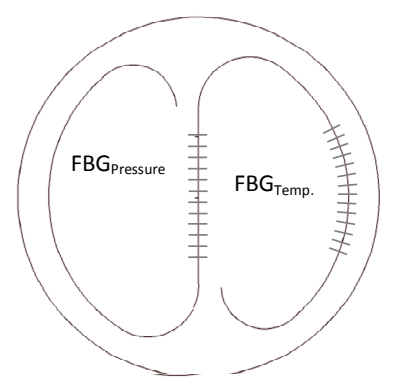

Figure 1. Schematic illustration of the circular membrane element of the fiber transducer.

\subsection{Design and fabrication}

The material for the membrane must accomplish specifications such as: it must be rigid enough to bear the pressure to be measured, it must not exhibit "memory effect" recovering the position when the cause changing the shape has ceased, and it must also be corrosion-resistant maintaining its physical and chemical properties over time. Options such as stainless steel and several alloys were analyzed.

Taking into account the feasibility to embed the FBG's during the membrane manufacturing process, a composite fiber reinforced plastic (FRP) was finally chosen for the membrane element. This ensures the avoidance of typical problems in bonding the FBG element to the materials and their reliability in working conditions. In addition, by embedding the FBGs into the material, higher sensitivities to temperature are obtained due to the higher expansion coefficient of the membrane material.

With the proposed design, different sensitivities of the two gratings and not proportional to each other are reached and hence the temperature and the pressure are properly discriminated by solving the following system of equations:

$$
\begin{aligned}
& \Delta \lambda_{F B G 1}=\Delta P \cdot K_{P 1}+\Delta T \cdot K_{T 1} \\
& \Delta \lambda_{F B G 2}=\Delta P \cdot K_{P 2}+\Delta T \cdot K_{T 2}
\end{aligned}
$$

where $K_{P 1}, K_{P 2}, K_{T 1}, K_{T 2}$ are the sensitivities of the pressure and temperature of each of the two diffraction grating elements. Different $K_{x y}$ coefficient values are obtained by placing the diffraction grating (FBG $\left.\mathrm{F}_{\text {Pressure }}\right)$ in the membrane center and the other $\left(\mathrm{FBG}_{\mathrm{Temp}}\right)$ slightly curved along the circular shape side of the membrane. The former shows the higher sensitivity to pressure due to be located at the center of the membrane.

The membrane was implemented in four steps: i) a sheet of fiber reinforced plastic without FBG embedded was manufactured; ii) the optical fiber with the two FBG would stand on the sheet in the proper positions; iii) new reinforced plastic layers are placed embedding both diffraction gratings and finally, iv) the circular membranes elements are obtained using a CNC machine.

To enable the easy installation of the proposed transducer in large diameter water pipes used in the fluid transportation a special holder was designed using a commercial element with the typical thread commonly employed. A custom holder was designed and constructed to obtain the easy integration of the transducer membrane. It allows that, while one side was at high pressure, the other one not, and no loss of liquid or gas was produced from one side to the other. In Figure 2 a cross-section of the transducer and a picture are shown.

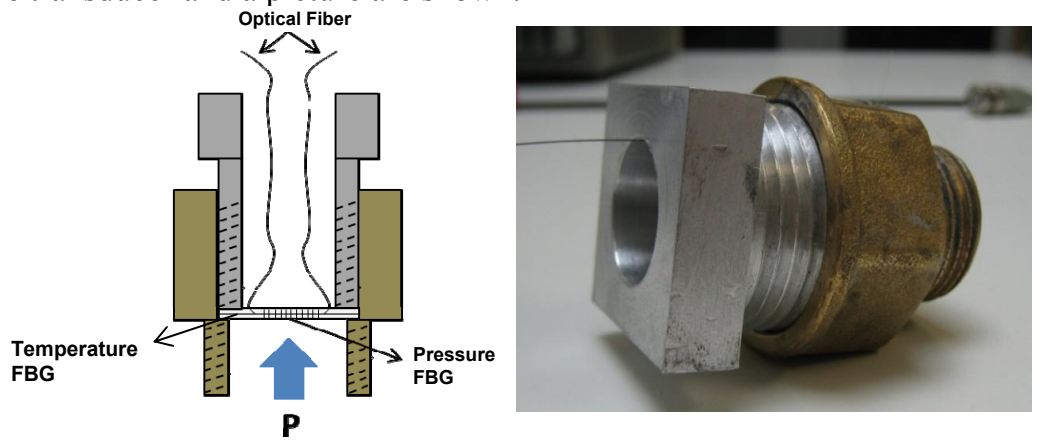

Figure 2. Schematic illustration of the transducer holder (left) and a picture of a preliminary prototype (right). 


\section{CHARACTERIZATION}

The transducer prototypes were characterized by using two different setups: one for temperature and another for temperature and pressure characterization, respectively.

\subsection{Temperature characterization}

The transducer prototypes were placed inside a climatic chamber, and outside a FBG interrogation unit (model Braggmetter from Fibersensing) has been used to interrogate the transducer FBG's optical response, both computer controlled. The measurements were carried out only when the temperature in the chamber matched the programed temperature step in steady state.

The experimental results for the two FBGs of one of the transducers, ranged from 0 to $25{ }^{\circ} \mathrm{C}$, are shown in Figure 3 . As shown in the figure, an excellent linearity and different slopes were obtained, as expected, from both FBGs of the transducer.

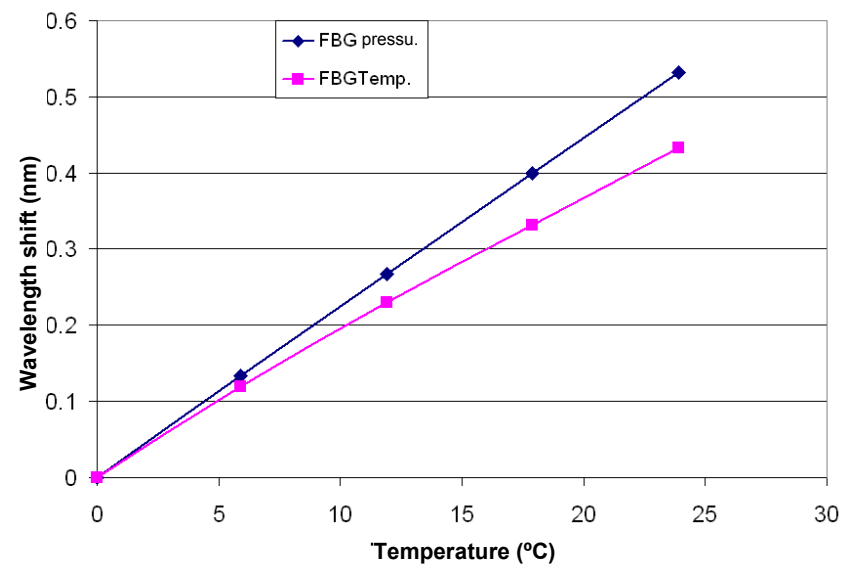

Figure 3. Response to the temperature of both FBGs embedded into the transducer membrane

\subsection{Pressure characterization}

For the pressure characterization the setup shown in Figure 4 has been implemented. The transducer was installed in a watertight pipe. The input and output of water is made by solenoid valves. A pump pressure is connected in the pipe input. The pressure was measured using a digital manometer, model LabDMM from AEP. The latter is calibrated and certified by the Italian accreditation organization Accredia. The same FBG interrogation unit used in the temperature setup has been employed, being all controlled by a computer. The measurements have been made using custom software. It controlled the open time of both solenoid valves in order to obtain the desired pressure within the pipe. The measurement of the Bragg wavelengths were performed when the pressure was stable, and afterwards another pressure was programed.

Scanning from 0 to 5.5 bar with steps of 0.2 bar have been carried out. The characterization has been made at constant environment temperature. The results obtained are shown in Figure 5. Both results are practically linear and the sensitivity for the FBG positioned in the center of the membrane is 10 times higher, as expected.

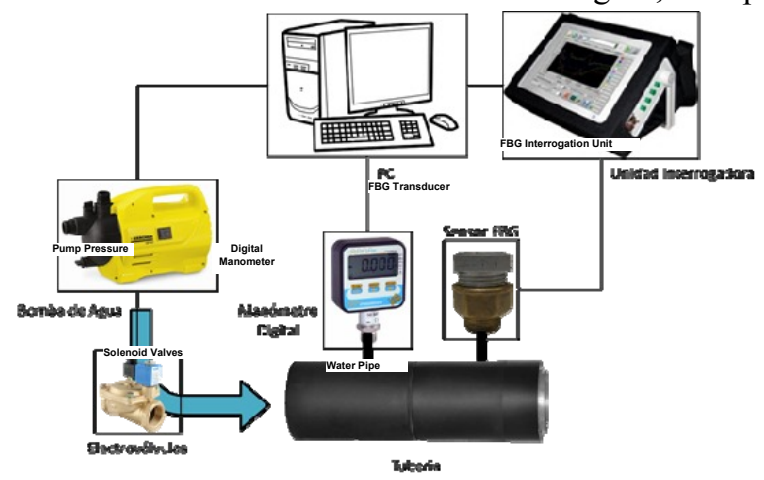

Figure 4. Scheme of the pressure experimental characterization setup. 


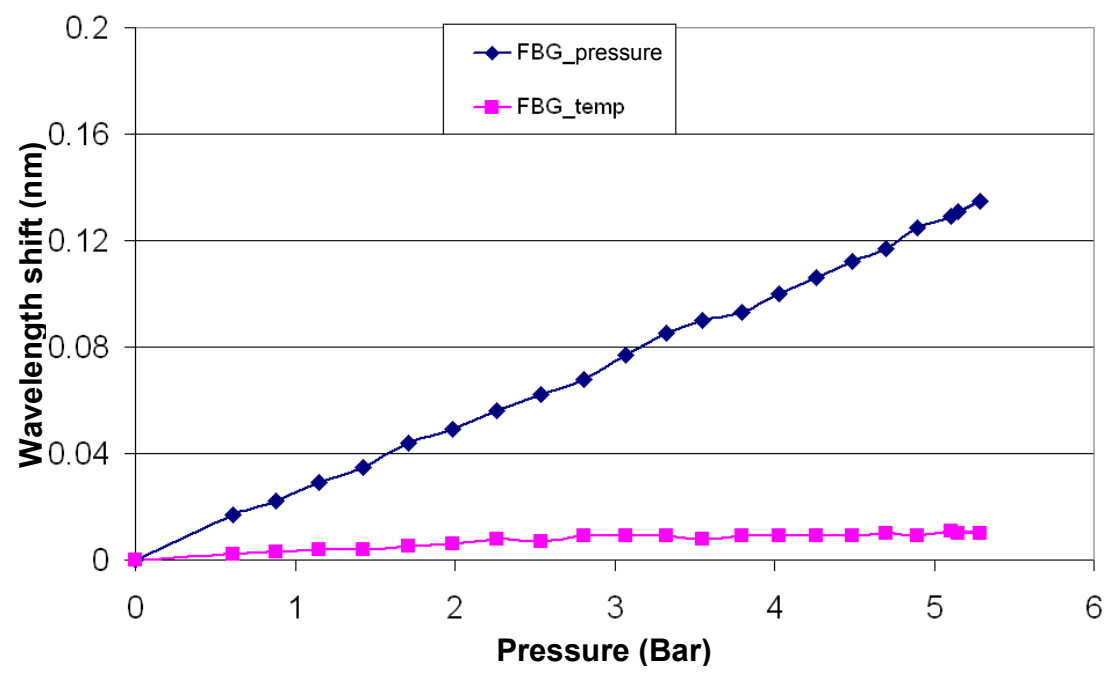

Figure 5. Response to the pressure of both FBGs embedded into the membrane.

\section{CONCLUSIONS}

The design, fabrication and experimental characterization of a pressure and temperature transducer have been presented in this paper. The transducer is based on a circular membrane of carbon fiber reinforced plastic (CFRP) with two embedded FBGs. Both gratings are arranged in the membrane to have different sensitivities to pressure and temperature, therefore it is possible to discriminate between these two parameters.

The membrane was placed in a custom holder designed to guarantee the sealing of the transducer between both sides of the membrane and to achieve different sensitivities to the pressure in booth FBGs.

The transducer has been characterized for both temperature and pressure. The behavior was practically linear in both cases and also with different sensitivities for both FBGs. The feasibility of the developed transducer to be used in real large diameter water pipes has been successfully checked in a field trial.

\section{ACKNOWLEDGEMENTS}

The authors would like to acknowledge the support provided by the Spanish CICYT through the TEC2010-20224-C0202 and the FASO (co-funded by Copsesa and the Cantabria Government) projects.

\section{REFERENCES}

[1] J. M. Lopez-Higuera, "Introduction to optical fiber sensor technology" book chapter 1. John Wiley \& Sons Inc., New York, 1 (2002).

[2] Manuel López-Amo and JM López-Higuera "Multiplexing Techniques for FBG Sensors"chapter in "Fiber Bragg Grating Sensors: Recent Advancements, Industrial Applications and Market Exploitation";Bentham Science Publishers Ltd., 2011.

[3] L. M.G. Xu, "Optical in-Fibre gratting high pressure sensor", Electronic letters Vol.29, No.4, 398-399, 1993.

[4] H. G. a. J. M.G. Xu, "Fibre Grating pressure sensor with enhanced sensitivity using a glass-bubble housing", Electronic Letters, vol. 32, nº 2, pp. 128-129, 1996.

[5] H. Z. Q. Z. Lihui Liu, "Temperature-independent FBG pressure sensor with high sensitivity”, Elsevier, vol. 13, pp. 78-80, 2007.Booth, N. and Smith, A. S., [Infrared Detectors], Goodwin House Publishers, New York \& Boston, 241-248 (1997).

[6] A. Quintela, J.M. Lázaro, M.A. Quintela, J. Mirapeix, V Muñoz-Berti, J.M. López-Higuera, “'Angle transducer based on fiber Bragg gratings able for tunnel auscultation'”, EWOFS'10, Oporto, Portugal, . 765318-1/7653184, 2010. 